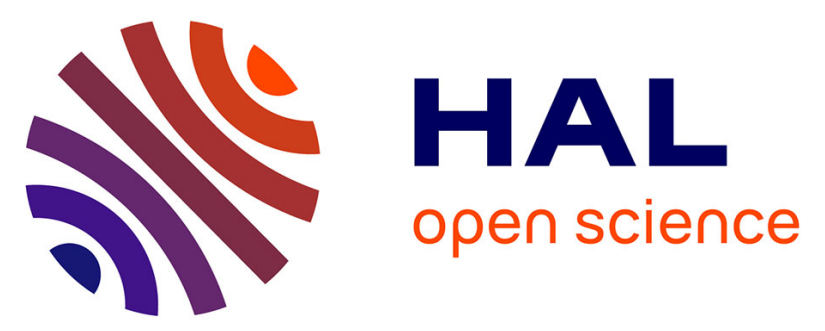

\title{
The symmetric sum-capacity of the Gaussian Half-Duplex Causal Cognitive Interference Channel to within a constant gap
}

Martina Cardone, Daniela Tuninetti, Raymond Knopp, Umer Salim

\section{- To cite this version:}

Martina Cardone, Daniela Tuninetti, Raymond Knopp, Umer Salim. The symmetric sumcapacity of the Gaussian Half-Duplex Causal Cognitive Interference Channel to within a constant gap. IEEE International Symposium on Information Theory, Jul 2013, Turkey. pp.754-758, 10.1109/ISIT.2013.6620327 . hal-00875022

\section{HAL Id: hal-00875022 \\ https://hal.science/hal-00875022}

Submitted on 20 Oct 2013

HAL is a multi-disciplinary open access archive for the deposit and dissemination of scientific research documents, whether they are published or not. The documents may come from teaching and research institutions in France or abroad, or from public or private research centers.
L'archive ouverte pluridisciplinaire HAL, est destinée au dépôt et à la diffusion de documents scientifiques de niveau recherche, publiés ou non, émanant des établissements d'enseignement et de recherche français ou étrangers, des laboratoires publics ou privés. 


\title{
The symmetric sum-capacity of the Gaussian Half-Duplex Causal Cognitive Interference Channel to within a constant gap
}

\author{
Martina Cardone $^{\dagger}$, Daniela Tuninetti*, Raymond Knopp ${ }^{\dagger}$, Umer Salim ${ }^{\ddagger}$ \\ $\dagger$ Eurecom, Campus SophiaTech, 450 Route des Chappes, Biot, 06410, Email: \{cardone, knopp\}@eurecom.fr \\ * University of Illinois at Chicago, Chicago, IL 60607, USA, Email: danielat@uic.edu \\ $\ddagger$ Intel Mobile Communications, Sophia Antipolis, 06560, France, Email: umer.salim@intel.com
}

\begin{abstract}
This paper studies the sum-capacity of the Gaussian half-duplex causal cognitive interference channel, a channel model with two transmitter-receiver pairs where a (cognitive) source cooperates with the other (primary) source in sending data through a shared channel. In contrast to the classical cognitive radio model, here the cognitive source can not transmit and receive at the same time and must causally learn the primary message through a noisy channel. Achievable strategies are developed and shown to match known upper bounds on the symmetric sum-capacity of this channel to within a constant gap for all values of channel parameters. In the process, the generalized degrees of freedom of the channel is characterized.
\end{abstract}

\section{INTRODUCTION}

We consider the Gaussian cognitive overlay channel characterized by two sources, namely PTx and CTx, and two destinations, namely PRx and CRx. Each source wishes to communicate with the corresponding receiver (PTx with PRx, CTx with CRx). PTx and PRx are deployed by the spectrum license owner and are referred to as the primary pair. CTx and CRx opportunistically use the licensed spectrum and are referred to as the cognitive pair. The classical information theoretic model for the cognitive overlay channel assumes that the CTx has a non-causal and perfect knowledge of the primary user's message prior to transmission [1]. This assumption has been very useful to show the potential fundamental gains of a cognitive radio technology. However, from a practical point of view, it might not be realistic. In this work we remove this ideal assumption by imposing causality constraints for the CTx. To this end, the CTx must learn the primary message over a lossy communication link between PTx and CTx. Moreover, we assume that the CTx operates in Half-Duplex (HD) mode, i.e., it can either transmit or receive at any given time, but not both. We shall denote this system as the Half-Duplex Causal Cognitive Interference Channel (HD-CCIC).

Related Work. The exact characterization of the capacity region for a general memoryless network is challenging. Recently, it has been advocated that progress can be made towards determining the capacity by showing that achievable strategies are provably close to computable outer bounds [2]. For the non-causal cognitive channel in Gaussian noise the capacity region has been exactly determined for some parameter regimes and it is known to within 1 bit otherwise [3].
This work considers the HD-CCIC, which can be studied within the framework of the Interference Channel (IC) with generalized feedback, or with bilateral Full-Duplex (FD) source cooperation [4], [5], [6], by imposing that only the CTx cooperates and by using the formalism of [7]. In [7], it was showed that there is no need to develop a separate theory for memoryless networks with HD nodes as the HD constraints can be incorporated into the memoryless FD framework by introducing a "state random variable" in the input definition. In [7], it was also proved that the largest rate can be achieved by randomly switching between the transmit- and receive-phases at the HD relay node in a simple relay channel. In this way, the randomness that lies into the switch can be used to transmit (at most 1 bit per channel use of) information to the destination. We shall refer to this specific HD mode of operation as random switch [7], as opposed to deterministic switch where the transmit- and receive-phases are predetermined.

Outer and inner bounds for the sum-capacity of the Gaussian IC with either FD source or destination bilateral cooperation were derived in [4]; in [8], a sum-rate outer bound for the general memoryless IC with source cooperation was derived by extending the work of [9]; in [5], a sum-rate outer-bound for a special class of "injective" IC with FD source cooperation was proposed by extending the work of [10]. The largest known achievable region for the general memoryless IC with FD source cooperation, to the best of our knowledge, is in [6]. In [5, Prop.3] the symmetric sum-capacity of the Gaussian IC with FD source cooperation was characterized to within 6 bits, and in [11] to within 2 bits in the "strong cooperation regime".

The Gaussian IC with bilateral and unilateral HD source cooperation was studied in [12] where the sum-capacity was characterized to within 20 bits and 31 bits, respectively, by considering deterministic switch at the CTx in both inner and outer bounds. In this work we overcome a number of limitations and improve on the results of [12] as follows: (i) we properly account for random switch at the CTx in the outer bound, (ii) we consider the classical definition of sumcapacity without introducing any 'back-off' in the PTx rate, (iii) we derive the generalized Degrees-of-Freedom (gDoF) in closed form rather than expressing it implicitly as the solution of a linear program, and (iv) we reduce the gap to 10.503 bits. 


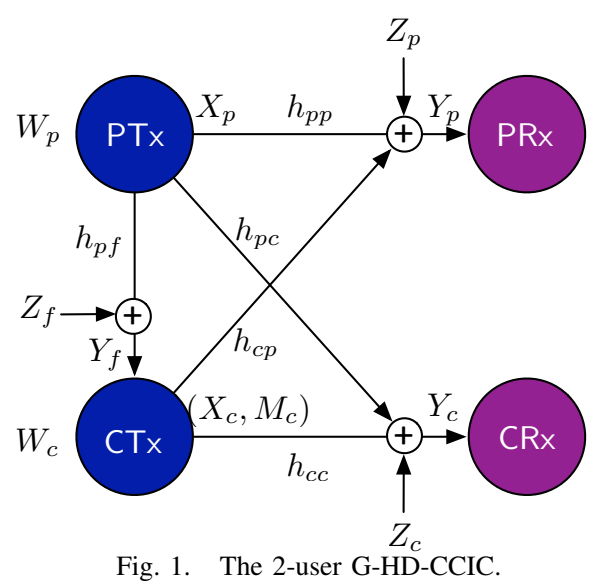

Contributions and Paper Outline. We focus on the symmetric Gaussian HD-CCIC, described in Section II, and characterize its gDoF in closed-form and its sum-capacity to within a constant gap in Section IV. In order to accomplish this we first specialize, in Section III, the sum-rate bounds of [4], [5], [8] to the HD-CCIC by using the formalism of [7]. We then develop achievable schemes that are asymptotically optimal for different parameter regimes. These schemes could possibly be obtained as a special case of [6]; due to the complexity of [6] we develop schemes inspired by the linear deterministic approximation of the Gaussian HD-CCIC in the spirit of [2] (not reported here for space limitations but available at [13]). An interesting feature of these schemes is that they employ successive decoding at the receivers, which is simpler than joint decoding. Moreover, our novel achievable schemes give insights on when causal cognitive radio might be of practical interest, i.e., when it outperforms the non-cooperative case, and it performs as bilateral cooperation.

\section{SySTEM MOdEL}

General Memoryless Channel Model. A CCIC is described by two input alphabets $\left(\mathcal{X}_{p}, \mathcal{X}_{c}\right)$, three output alphabets $\left(\mathcal{Y}_{f}, \mathcal{Y}_{p}, \mathcal{Y}_{c}\right)$ and a memoryless transition probability $P_{Y_{f}, Y_{p}, Y_{c} \mid X_{p}, X_{c}}$. PTx aims to transmit a message $W_{p} \in[1$ : $\left.2^{N R_{p}}\right]$ to PRx and CTx wishes to send a message $W_{c} \in$ $\left[1: 2^{N R_{c}}\right]$ to $\mathrm{CRx}$, where $N$ denotes the codeword length and $R_{p}$ and $R_{c}$ are the transmission rates for PTx and CTx, respectively, measured in bits per channel use (logarithms are in base 2). The messages $W_{p}$ and $W_{c}$ are independent and uniformly distributed on their respective domains. At time $i \in[1: N]$ PTx encodes its message $W_{p}$ into $X_{p, i}\left(W_{p}\right)$ and CTx maps its message $W_{c}$ and its past channel observations into $X_{c, i}\left(W_{c}, Y_{f}^{i-1}\right)$. At time $N$, PRx estimates its intended message $W_{p}$ based on all its channel observations as $\widehat{W}_{p}\left(Y_{p}^{N}\right)$, and similarly CRx outputs $\widehat{W}_{c}\left(Y_{c}^{N}\right)$. The capacity region is defined as the convex closure of all non-negative rate pairs $\left(R_{p}, R_{c}\right)$ such that $\max _{u \in\{c, p\}} \mathbb{P}\left[\widehat{W}_{u} \neq W_{u}\right] \rightarrow 0$ as $N \rightarrow+\infty$.

Gaussian HD-CCIC (G-HD-CCIC). HD channels represent a special case of the memoryless FD framework [7]. With a slight abuse of notation compared to the previous paragraph, let the channel input at the CTx be the pair $\left(X_{c}, M_{c}\right)$, where
$X_{c} \in \mathcal{X}_{c}$ as before and $M_{c} \in\{0,1\}$ is the state random variable that indicates whether the CTx is in receive-mode $\left(M_{c}=0\right)$ or in transmit-mode $\left(M_{c}=1\right)$. The single-antenna G-HD-CCIC, in Fig. 1, has the input/output relationship

$$
\left[\begin{array}{c}
Y_{f} \\
Y_{p} \\
Y_{c}
\end{array}\right]=\left[\begin{array}{ccc}
1-M_{c} & 0 & 0 \\
0 & 1 & 0 \\
0 & 0 & 1
\end{array}\right] \mathbf{H}\left[\begin{array}{cc}
1 & 0 \\
0 & M_{c}
\end{array}\right]\left[\begin{array}{l}
X_{p} \\
X_{c}
\end{array}\right]+\left[\begin{array}{c}
Z_{f} \\
Z_{p} \\
Z_{c}
\end{array}\right],
$$

where the inputs are subject to the average power constraints $\mathbb{E}\left[\left|X_{i}\right|^{2}\right] \leq 1, i \in\{p, c\}$, and $\mathbf{H}$ is the constant complexvalued channel matrix that defines the connectivity of the network defined in the symmetric case as

$$
\mathbf{H}:=\left[\begin{array}{cc}
h_{p f} & \star \\
h_{p p} & h_{c p} \\
h_{p c} & h_{c c}
\end{array}\right]=\left[\begin{array}{cc}
\sqrt{C} & \star \\
\sqrt{S} & \sqrt{I} \mathrm{e}^{\mathrm{j} \theta_{1}} \\
\sqrt{I} \mathrm{e}^{\mathrm{j} \theta_{2}} & \sqrt{S}
\end{array}\right],
$$

where $\star$ indicates the channel gain that does not affect the capacity region (because CTx can remove its transmitted signal $X_{c}$ from its channel output $Y_{f}$ ) and where some channel gains can be taken to be real-valued and non-negative because a node can compensate for the phase of one of its channel gains. The noises are proper-complex Gaussian random variables with parameter $Z_{k} \sim \mathcal{N}(0,1), k \in\{f, p, c\}$. In the following we assume that the noises are independent.

Performance metrics. The capacity of the channel in (1) is unknown. Here we make progress by first determining its gDoF and then showing that the sum-capacity outer-bound is achievable to within a constant gap for any value of the channel parameters. The gDoF of a symmetric Gaussian noise channel is defined as [10]

$$
\mathrm{d}(\alpha, \beta):=\lim _{S \rightarrow+\infty} \frac{\max \left\{R_{p}+R_{c}\right\}}{2 \log (1+S)},
$$

where the maximization is over all achievable rate pairs $\left(R_{c}, R_{p}\right)$ and where, for some fixed non-negative $(\alpha, \beta)$, we parameterized $C=S^{\beta}$ and $I=S^{\alpha}$. Here $S$ represents the SNR on the direct links.

The gDoF region gives an asymptotically exact characterization of the capacity at infinite SNR. At finite SNR the sum-capacity is said to be known to within GAP bits if we can show an inner bound $\left(R_{p}+R_{c}\right)^{(\mathrm{IB})}$ and an outer bound $\left(R_{p}+R_{c}\right)^{(\mathrm{OB})}$ such that

$$
0 \leq\left(R_{p}+R_{c}\right)^{(\mathrm{OB})}-\left(R_{p}+R_{c}\right)^{(\mathrm{IB})} \leq \mathrm{GAP}
$$

for all channel parameters $\left(S, C, I, \theta_{1}, \theta_{2}\right)$.

\section{OUTER BOUNDS}

In the literature several outer-bounds are known for bilateral source cooperation, where each source works in FD mode [4], [5], [8]. Here we specialize them for the case of HD unilateral cooperation by following [7]. In particular, we proceed through the following steps: (i) in the outer bounds for the general memoryless channel with input $X_{c}$, we substitute $X_{c}$ with the pair $\left(X_{c}, M_{c}\right)$; (ii) we apply the mutual information chain rule so as for any triplet of random variables $(A, B, C)$ we have the bound $I\left(A, X_{c}, M_{c} ; B \mid C\right) \leq H\left(M_{c}\right)+I\left(A, X_{c} ; B \mid C, M_{c}\right)$; 


$$
\begin{aligned}
& R_{p}+R_{c} \leq \min \left\{\left(R_{p}+R_{c}\right)^{(\mathrm{OB}-\mathrm{CS})},\left(R_{p}+R_{c}\right)^{(\mathrm{OB}-\mathrm{DT})},\left(R_{p}+R_{c}\right)^{(\mathrm{OB}-\mathrm{PV})}\right\} \\
& \left(R_{p}+R_{c}\right)^{(\mathrm{OB}-\mathrm{CS})}:=2.507+\gamma \log (1+S+C)+2(1-\gamma) \log (1+S) \\
& \left(R_{p}+R_{c}\right)^{(\mathrm{OB}-\mathrm{DT})}:=3+\gamma \log (1+S)+(1-\gamma) \log \left(\frac{\max \{I, S\}}{I}\right)+(1-\gamma) \log \left(1+(\sqrt{S}+\sqrt{I})^{2}\right) \\
& \left(R_{p}+R_{c}\right)^{(\mathrm{OB}-\mathrm{PV})}:=5.503+\gamma \log (1+S+C+I)+2(1-\gamma) \log \left(1+I+\frac{S+2 \sqrt{I S}}{1+I}\right) .
\end{aligned}
$$

this follows since for a binary-valued random variable $M_{c}$ we have $I\left(M_{c} ; B \mid C\right) \leq H\left(M_{c}\right)=\mathcal{H}(\gamma)$ for some $\gamma:=\mathbb{P}\left[M_{c}=\right.$ $0] \in[0,1]$ and where $\mathcal{H}(\gamma)$ is the binary entropy defined as $\mathcal{H}(\gamma)=-\gamma \log (\gamma)-(1-\gamma) \log (1-\gamma)$; (iii) for all the remaining mutual information terms, which are conditioned on $M_{c}$, the 'Gaussian maximizes entropy'-principle [14, Appendix 16A] guarantees that we can restrict attention to joint Gaussian inputs with covariance matrix

$$
\left.\operatorname{Cov}\left[\begin{array}{l}
X_{p} \\
X_{c}
\end{array}\right]\right|_{M_{c}=\ell}=\left[\begin{array}{ccc}
P_{p, \ell} & \rho_{\ell} & \sqrt{P_{p, \ell} P_{c, \ell}} \\
\rho_{\ell}^{*} & \sqrt{P_{p, \ell} P_{c, \ell}} & P_{c, \ell}
\end{array}\right]:\left|\rho_{\ell}\right| \leq 1
$$

for $\ell \in\{0,1\}$ and for some $\left(P_{p, 0}, P_{p, 1}, P_{c, 0}, P_{c, 1}\right) \in \mathbb{R}_{+}^{4}$ satisfying the average power constraint

$$
\gamma P_{u, 0}+(1-\gamma) P_{u, 1} \leq 1, u \in\{p, c\} ;
$$

(iv) we rewrite the average power constraint as follows: since PTx transmits in both states $M_{c}=0$ and $M_{c}=1$ we define, for some $\beta \in[0,1], P_{p, 0}=\frac{\beta}{\gamma}$ and $P_{p, 1}=\frac{1-\beta}{1-\gamma}$; since the CTx transmission only affects the receivers output when $M_{c}=1$, we let $P_{c, 0}=0$ and $P_{c, 1}=\frac{1}{1-\gamma} ;(\mathrm{v})$ finally, the upper bounds in (5) (obtained from the cut-set bound [4, eq.(81)+eq.(82)]), in (6) (obtained from [8, eq.(4d)]) and in (7) (obtained from [5, eq.(6-7)]), given at the top of this page, are obtained by upper bounding each individual mutual information term over $\left(\rho_{0}, \rho_{1}, \beta\right) \in[0,1]^{3}$, for a fixed $\gamma$; (vi) after these steps, each outer bound has terms that are linear combinations of terms of the type ' $\gamma \log \left(\frac{1}{\gamma}\right)$ '; by numerically optimizing these quantities, we obtain the constants that characterize the outer bounds in (5), (6) and (7).

\section{GDOF AND SUM-CAPACITY TO WITHIN A CONSTANT}

Our first main results is:

\section{Theorem 1 The gDoF of the symmetric G-HD-CCIC is}

$$
\begin{gathered}
\mathrm{d}(\alpha, \beta) \leq \frac{1}{2} \max _{\gamma} \min \{\gamma \max \{1, \beta\}+2(1-\gamma), \\
\gamma+(1-\gamma)\left(\max \{1, \alpha\}+[1-\alpha]^{+}\right), \\
\gamma \max \{\alpha, \beta, 1\}+(1-\gamma) \max \{2 \alpha, 2-2 \alpha\}\} \\
= \begin{cases}1-\alpha+\frac{1}{2} \frac{[\beta-2+2 \alpha]^{+} \alpha}{\beta+\alpha-1} & \alpha \in[0,1 / 2) \\
\alpha+\frac{1}{2} \frac{[\beta-2 \alpha]^{+}(2-3 \alpha)}{\beta-3 \alpha+1} & \alpha \in[1 / 2,2 / 3) \\
1-\frac{1}{2} \alpha & \alpha \in[2 / 3,1) \\
\frac{1}{2} \alpha & \alpha \in[1,2) \\
1+\frac{1}{2} \frac{[\beta-2]^{+}(\alpha-2)}{\beta+\alpha-3} & \alpha \in[2, \infty) .\end{cases}
\end{gathered}
$$

Proof: The proof that (8d) upper bounds the $\mathrm{gDoF}$ follows by substituting the sum-rate upper bounds in (5), (6) and (7) into the gDoF definition in (3). The proof that (8d) is achievable is given in the Appendix.

Fig. 2 shows the optimal $\mathrm{gDoF}$ and the gap for the symmetric G-HD-CCIC. The whole set of parameters has been partitioned into multiple sub-regions depending upon different levels of cooperation $(\beta)$ and interference $(\alpha)$ strengths.

The $\mathrm{gDoF}$ in $(8 \mathrm{~d})$, in the different interference regimes (i.e., different values of $\alpha$ ), is the sum of the gDoF without cooperation (obtained for $\beta=0$ ) and a term that is strictly positive only for sufficiently large $\beta$. In particular, $\beta$ must be larger than the gDoF without cooperation. This can be explained as follows:

- In weak interference $\alpha \in[0,2 / 3)$ and without cooperation $\beta=0$ the PV bound in (8c) is the tightest [10]. The PV bound in $(8 \mathrm{c})$ is optimized by $\gamma=0$, which is equivalent to nocooperation, whenever $\max \{1, \alpha, \beta\} \leq 2 \max \{1-\alpha, \alpha\}=$ $2 \mathrm{~d}^{\text {(NoCoop) }}(\alpha) \Longleftrightarrow \beta \leq 2 \mathrm{~d}^{\text {(NoCoop) }}(\alpha)$. Thus, unilateral HD cooperation is beneficial in terms of $\mathrm{gDoF}$ only when $\beta$ is larger than the gDoF without cooperation. In this case, the optimal $\gamma$, obtained by equating the PV bound in $(8 \mathrm{c})$ to the DT bound in $(8 b)$, is

$$
\gamma^{(\mathrm{PV} . \mathrm{DT})}=\frac{\min \{2-3 \alpha, \alpha\}}{\min \{2-3 \alpha, \alpha\}+\beta-1},
$$

which then leads to the first two cases in (8d).

- In very strong interference $\alpha \geq 2$ and without cooperation $\beta=0$ the CS bound in (8a) is the tightest [9]. The CS bound in (8a) is optimized by $\gamma=0$, which is equivalent to nocooperation, whenever $\max \{1, \beta\} \leq 2=2 \mathrm{~d}^{\text {(NoCoop) }}(\alpha) \Longleftrightarrow$ $\beta \leq 2 \mathrm{~d}^{(\mathrm{NoCoop})}(\alpha)$. Again we see that unilateral HD cooperation is beneficial in terms of $\mathrm{gDoF}$ only when $\beta$ is larger than the $\mathrm{gDoF}$ without cooperation. In this case, the optimal $\gamma$, obtained by equating the CS bound in (8c) to the DT one in $(8 b)$, is

$$
\gamma^{(\mathrm{CS} . \mathrm{DT})}=\frac{\alpha-2}{\beta+\alpha-3},
$$

which then leads to the last case in $(8 \mathrm{~d})$.

- In moderately-weak and strong interference $\alpha \in[2 / 3,2)$ and with no cooperation $\beta=0$ the DT bound in (8b) is the tightest [9]. This bound is always optimized by $\gamma=0$, which is equivalent to no-cooperation. Hence in this regime it is always gDoF-optimal to operate the channel as a pure IC.

Summarizing the above results we have:

- In regions $1,2,4,5,6,7$ and 9 in Fig. 2, unilateral HD 


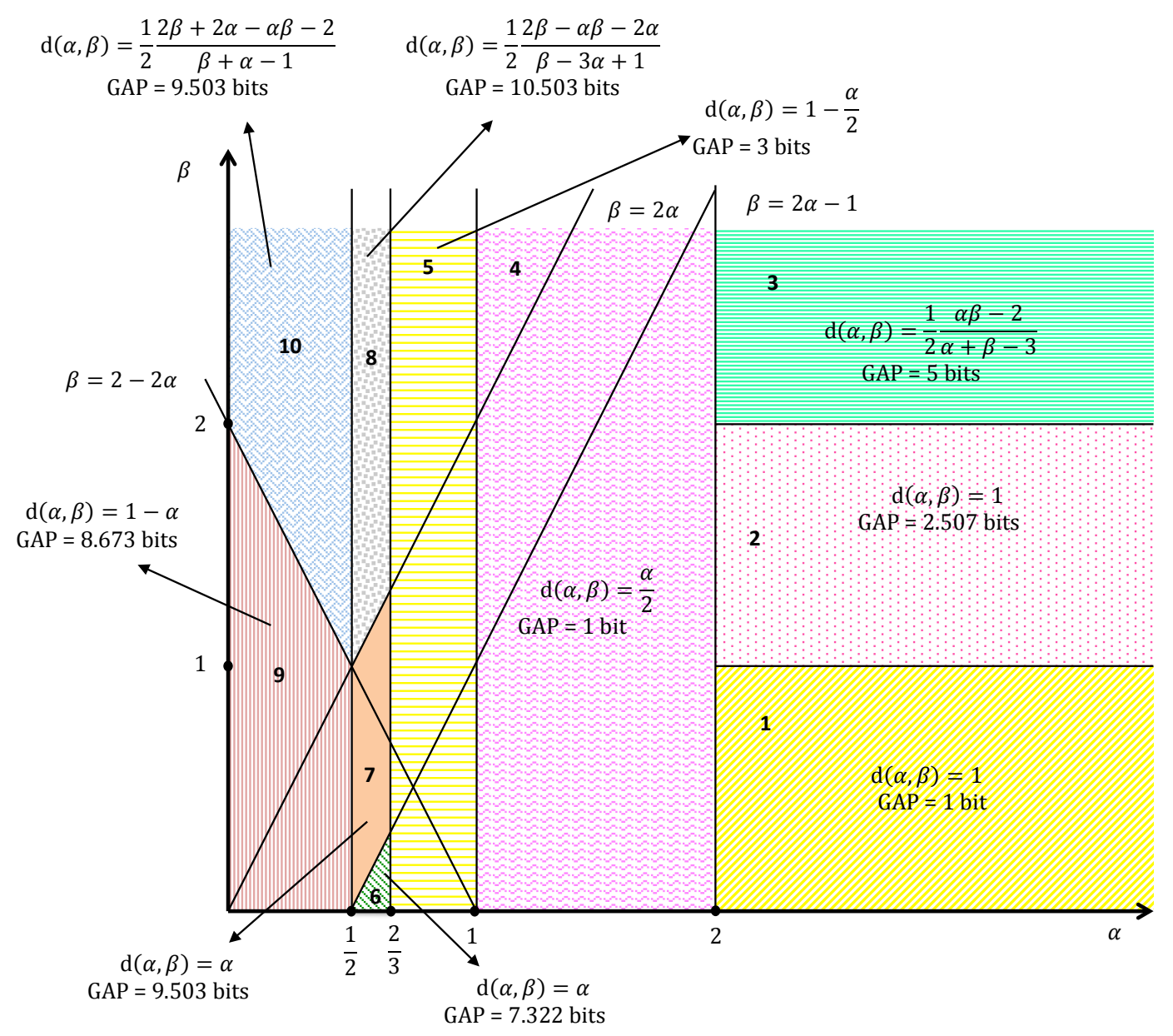

Fig. 2. Optimal gDoF and constant gap for the symmetric G-HD-CCIC in the different regimes of $(\alpha, \beta)$.

cooperation might not be worth implementing in practical systems since the same $\mathrm{gDoF}$ is achieved without cooperation. - In regions $1,4,5$ and 6 the $\mathrm{gDoF}$ is equal to the one with unilateral FD cooperation [15]. This implies that, in these regimes, the same gap results found for the FD case in [15] hold in the case of unilateral HD cooperation. In these regimes there is no penalty in HD cooperation.

- By computing the gDoF of the IC with bilateral HD source cooperation [16] we see that in regions $1,2,6,7,9$, part of 5 (with $\beta \leq 3-2 \alpha$ ) and part of 4 (with $\beta \leq 2 \alpha-1$ ), the $\mathrm{gDoF}$ are the same as those computed in the unilateral case. Thus, for this set of parameters, unilateral cooperation attains the same gDoF of bilateral cooperation but with less resources and therefore represents a better trade-off in practical systems.

Our second main result is:

Theorem 2 The sum-rate upper bound in (4) is achievable to within 10.503 bits for the symmetric G-HD-CCIC.

Proof: The proof can be found in the Appendix.

Before concluding we make some more remarks on the gap: - Most of our proposed schemes only use superposition coding. The only exception is in weak interference, i.e., regions 8 and 10 in Fig. 2, where we were not able to characterize the sum-rate to within a constant gap with superposition only.

- All the achievable schemes presented here use successive decoding at the receivers. This form of decoding, in practice, is simpler than joint decoding; thus our schemes may be used as guidelines to deploy practical cognitive radio systems.

- The gap computed in this work is around 3 bits bigger than the one computed in the FD case [15]. Possible ways to reduce this gap may be: (i) apply joint decoding at the receivers, (ii) develop block Markov schemes instead of taking inspiration by the linear deterministic approximation channel of the Gaussian noise channel at high SNR, (iii) develop achievable schemes that exploit the randomness in the switch to convey further useful information, (iv) develop tighter upper bounds than those used in this work, especially in weak interference where the gap is quite large.

\section{Conclusions}

In this work we considered the half-duplex causal cognitive channel. Differently from the original overlay cognitive model, in which the CTx has a full a priori knowledge of the message of the PTx, we consider a more practical scenario in which the CTx learns this message through a noisy link and 


$$
\begin{aligned}
\left(R_{p}+R_{c}\right)^{(\mathrm{IB} 1)}= & \gamma^{*} \log (1+\mathrm{S})-\gamma^{*} \log \left(1+\frac{\mathrm{S}}{1+\mathrm{S}}\right)+\gamma^{*} \log \left(1+\frac{\mathrm{C}}{1+\mathrm{S}}\right) \\
& +\left(1-\gamma^{*}\right) \log (1+\mathrm{S})+\left(1-\gamma^{*}\right) \log (1+\mathrm{S})-\left(1-\gamma^{*}\right) \log \left(1+\frac{\mathrm{S}}{1+\mathrm{S}}\right), \\
\left(R_{p}+R_{c}\right)^{(\mathrm{IB} 2)}= & \gamma^{*} \log (1+\mathrm{S})-\gamma^{*} \log \left(1+\frac{\mathrm{S}}{1+\mathrm{S}}\right)+\gamma^{*} \log \left(1+\frac{\mathrm{C}}{1+\mathrm{S}}\right) \\
& +2\left(1-\gamma^{*}\right) \log \left(1+\mathrm{I}+\frac{\mathrm{S}}{1+\mathrm{I}}\right)-2\left(1-\gamma^{*}\right) \log \left(1+\frac{\mathrm{I}}{1+\mathrm{I}}\right) \\
& +\left(1-\gamma^{*}\right) \log \left(1+\frac{\mathrm{S}}{1+\mathrm{I}}+\frac{\mathrm{I}}{1+\mathrm{I}}\right)-\left(1-\gamma^{*}\right) \log \left(1+\frac{\mathrm{SI}+\mathrm{I}+\mathrm{I}^{2}}{(1+\mathrm{I})^{2}}+\frac{\mathrm{S}}{1+\mathrm{I}}\right), \\
\left(R_{p}+R_{c}\right)^{(\mathrm{IB} 3)}= & \gamma^{*} \log (1+\mathrm{S})-\gamma^{*} \log \left(1+\frac{\mathrm{S}}{1+\mathrm{S}}\right)+\gamma^{*} \log \left(1+\frac{\mathrm{C}}{1+\mathrm{S}}\right)-\left(1-\gamma^{*}\right) \log (1+\mathrm{I}) \\
& +\left(1-\gamma^{*}\right) \log \left(1+\mathrm{I}+\frac{\mathrm{S}}{1+\mathrm{I}}\right)+\left(1-\gamma^{*}\right) \log \left(1+\frac{\mathrm{I}}{1+\mathrm{I}}+\mathrm{S}\right)-\left(1-\gamma^{*}\right) \log \left(1+\frac{\mathrm{I}}{1+\mathrm{I}}\right) .
\end{aligned}
$$

operates in HD mode. We derived the gDoF of the channel and characterized its symmetric sum-capacity to within a constant gap. This has been accomplished by developing novel achievable schemes for the different parameter regimes.

\section{ACKNOWLEDGMENT}

The work of D. Tuninetti was partially funded by NSF under award number 0643954 and thanks to the generous support of Telecom-ParisTech, Paris France, while the author was on a sabbatical leave at the same institution. Eurecom's research is partially supported by its industrial partners: BMW Group Research \& Technology, IABG, Monaco Telecom, Orange, SAP, SFR, ST Microelectronics, Swisscom and Symantec.The research carried out at Eurecom leading to these results has received funding from the EU Celtic+ Framework Program Project SPECTRA. The research at IMC leading to these results has received funding from the EU FP7 grant agreement iJOIN n $n^{\circ} 317941$.

\section{APPENDIX}

We develop achievable schemes inspired by the linear deterministic approximation of the G-HD-CCIC at high SNR. The details of the derivation may be found in [13].

- Region 1 ( $\alpha \geq 2$ and $\beta \leq 1$ ), Region 2 ( $\alpha \geq 2$ and $1<\beta \leq 2)$, Region $4(1 \leq \alpha<2)$, Region $5\left(\frac{2}{3} \leq \alpha<1\right)$, Region $6\left(\frac{1}{2} \leq \alpha<\frac{2}{3}\right.$ and $\left.\beta \leq 2 \alpha-1\right)$, Region 7 ( $\frac{1}{2} \leq \alpha<\frac{2}{3}$ and $2 \alpha-1<\beta \leq 2 \alpha)$ and Region 9 ( $\alpha<\frac{1}{2}$ and $\beta \leq 2-2 \alpha$ ). In these regions the gDoF upper bound equals that of the classical IC. Therefore an achievable scheme is given by the classical IC. The gap is at most of GAP $\leq 9.503$ bits.

- Region 3: $\alpha \geq 2$ and $\beta>2$. With the achievable scheme in [13, Appendices D-A, D-B], i.e., a two-phase strategy with superposition coding and successive decoding, we can achieve $\left(R_{p}+R_{c}\right)^{(\mathrm{IB} 1)}$ at the top of the page, where the limit behavior of $\gamma^{*}$ is the one in (10). The tightest upper bounds are those in (5) and in (6). The gap is GAP $\leq 5$ bits.

- Region 8: $\frac{1}{2} \leq \alpha<\frac{2}{3}$ and $\beta>2 \alpha$. With the achievable scheme in [13, Appendices D-A, D-C], i.e., a two-phase strategy with superposition coding, dirty-paper-coding and successive decoding, we can achieve $\left(R_{p}+R_{c}\right)^{(\mathrm{IB} 2)}$ at the top of the page, where the limit behavior of $\gamma^{*}$ is the one in (9). The tightest upper bounds are those in (6) and in (7). The gap is GAP $\leq 10.503$ bits.

- Region 10: $\alpha<\frac{1}{2}$ and $\beta>2-2 \alpha$. With the achievable scheme in [13, Appendices D-A, D-D], a two-phase strategy with superposition coding, dirty-paper-coding and successive decoding, we can achieve $\left(R_{p}+R_{c}\right)^{(\mathrm{IB} 3)}$ at the top of the page, where the limit behavior of $\gamma^{*}$ is the one in (9). The tightest upper bounds are those in (6) and in (7). The gap is GAP $\leq 9.503$ bits.

\section{REFERENCES}

[1] N. Devroye, P. Mitran, and V. Tarokh, "Achievable rates in cognitive radio channels," IEEE Trans. on Info. Theory, vol. 52, no. 5, May 2006.

[2] A. S. Avestimehr, Wireless network information flow: a deterministic approach, Ph.D. thesis, EECS Department, UCB, Oct 2008.

[3] S. Rini, D. Tuninetti, and N. Devroye, "Inner and outer bounds for the gaussian cognitive interference channel and new capacity results," IEEE Trans. on Info. Theory, vol. 58, no. 2, Feb. 2012.

[4] A. Host-Madsen, "Capacity bounds for cooperative diversity," IEEE Trans. on Info. Theory, vol. 52, no. 4, Apr. 2006.

[5] V.M. Prabhakaran and P. Viswanath, "Interference channels with source cooperation," IEEE Trans. on Info. Theory, vol. 57, no. 1, Jan. 2011.

[6] S. Yang and D. Tuninetti, "Interference channel with generalized feedback (a.k.a. with source cooperation): Part i: Achievable region," IEEE Trans. on Info. Theory, vol. 57, no. 5, May 2011.

[7] G. Kramer, "Models and theory for relay channels with receive constraints," in Allerton 2004, Sept. 2004.

[8] D. Tuninetti, "An outer bound region for interference channels with generalized feedback," in ITA 2010, Feb. 2010.

[9] G. Kramer, "Outer bounds on the capacity of gaussian interference channels," IEEE Trans. on Info. Theory, vol. 20, no. 3, 2004.

[10] R.H. Etkin, D.N.C. Tse, and Hua Wang, "Gaussian interference channel capacity to within one bit," IEEE Trans. on Info. Theory, vol. 54, no. 12, Dec. 2008.

[11] S. Yang and D. Tuninetti, "Interference channels with source cooperation in the strong cooperation regime: Symmetric capacity to within 2 bits/s/hz with dirty paper coding," in ASILOMAR 2011, Nov. 2011.

[12] R. Wu, Interference Channels with Half-Duplex Source Cooperation, Ph.D. thesis, Electrical and Computer Eng. Dept., UIUC, May 2011.

[13] M. Cardone, D. Tuninetti, R. Knopp, and U. Salim, "On the gaussian interference channel with half-duplex causal cognition," arxiv/1304.0604; submitted to IEEE JSAC Cognitive Radio Series, 2013.

[14] A. El Gamal and Y.H. Kim, Network Information Theory, Cambridge Univ. Press,, Cambridge U.K., 2011.

[15] M. Cardone, D. Tuninetti, R. Knopp, and U. Salim, "On the gaussian interference channel with causal cognition, or with unilateral source cooperation," arxiv/1207.5319; submitted to IEEE Trans. on Info. Theory, 2012.

[16] M. Cardone, D. Tuninetti, R. Knopp, and U. Salim, "Approximate sumcapacity of full-and half-duplex asymmetric interference channels with unilateral source cooperation," in ITA 2013, Feb. 2013. 